\title{
Cooperación de profesionales de las bibliotecas de Ciencias de la Salud como respuesta a la pandemia de la Covid-19
}

\section{Cooperation among health science library professionals in response to the Covid-19 pandemic}

\author{
María-Isabel Domínguez-Aroca; Grupo \#AyudaBiblioteca
}

Cómo citar este artículo:

Domínguez-Aroca, María-Isabel; Grupo \#AyudaBiblioteca (2020). “Cooperación de profesionales de las bibliotecas de Ciencias de la Salud como respuesta a la pandemia de la Covid-19". Profesional de la información, v. 29, n. 4, e290430.

https://doi.org/10.3145/epi.2020.jul.30

Artículo recibido el 18-04-2020 Aceptación definitiva: 19-05-2020

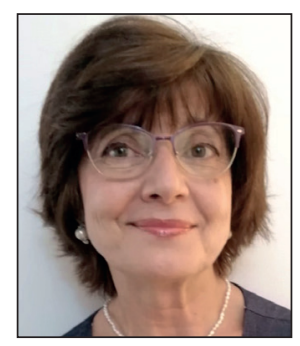
María-Isabel Domínguez-Aroca $\bowtie$ https://orcid.org/0000-0003-4237-1240
Universidad de Alcalá, CRAI-Biblioteca Edificio Cisneros.
Plaza de S. Diego, $\mathrm{s} / \mathrm{n}$.
28801 Alcalá de Henares (Madrid), España misabel.dominguez@uah.es

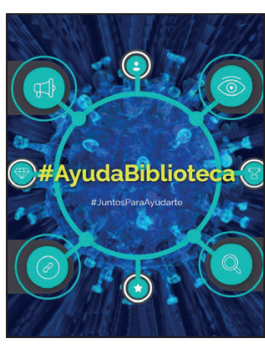

\section{Grupo \#AyudaBiblioteca}

España

ayuda.biblioteca.ccss@gmail.com

\section{Resumen}

La pandemia de la Covid-19 ha supuesto notables cambios en nuestra sociedad, incorporando nuevas formas de relación y de trabajo. Ha afectado especialmente a las profesiones del sistema sanitario, del que forman parte los bibliotecarios de Ciencias de la Salud, encargados de proporcionar información actualizada y de calidad a los sanitarios al frente de esta lucha. En este artículo se presenta el trabajo del colectivo de profesionales de bibliotecas de Ciencias de la Salud españolas \#AyudaBiblioteca, organizados para colaborar y dar una mejor respuesta a las necesidades informativas sobre la Covid-19. Cuenta con 90 participantes mayoritariamente de bibliotecas de hospitales, y también de organismos de investigación, sociedades científicas, colegios profesionales, consejerías, universidades, agencias de evaluación de tecnologías sanitarias y profesionales independientes. Como medio de comunicación se ha utilizado WhatsApp y como depósito de documentación Google Drive. Los recursos se visualizan en una web pública, y las estrategias de búsqueda se vuelcan en Diigo. Se utiliza Twitter con los hashtags \#AyudaBiblioteca y \#COVID19 como medio de comunicación externa para recibir y difundir información. Esta colaboración ha permitido aunar fuerzas en la búsqueda y recopilación coordinada de información fiable, basada en evidencia, contrastada y de calidad, y demuestra los beneficios del teletrabajo y de la colaboración online. También refleja las ventajas de la ciencia abierta y cómo mejora la investigación. Esta iniciativa muestra la importancia de los bibliotecarios de Ciencias de la Salud como apoyo al personal sanitario, académico y de investigación.

\section{Agradecimientos}

A todos los profesionales del grupo de bibliotecas de Ciencias de la Salud de España \#AyudaBiblioteca \#COVID19, que están colaborando en este proyecto, a los que de una forma especial han colaborado en la revisión de este artículo $^{1}$; sin todos ellos, no hubiese sido posible; y a las editoriales que han puesto todo el material sobre Covid-19 en acceso abierto.

También deseamos que sirva para agradecer a los trabajadores de las instituciones sanitarias de España y a todas las personas que están trabajando para que el resto estemos lo mejor posible. 


\section{Palabras clave}

Bibliotecas de Ciencias de la Salud; Bibliotecas de hospitales, Bibliotecas especializadas; Cooperación bibliotecaria; Bibliotecas universitarias; Bibliotecas de colegios profesionales; Centros de documentación; Bibliotecarios; Documentalistas; Profesionales de la información; Difusión de la información; Innovación bibliotecaria; Teletrabajo; Acceso abierto; Covid-19; Coronavirus; Pandemias.

\section{Abstract}

The Covid-19 pandemic has brought about notable changes in our society, including new forms of relationships and work. It has especially affected the professions related to the health system, which includes health science librarians, who are responsible for providing up-to-date and high-quality information to health workers on the front line of this fight. This article presents the work of a group of professionals working in Spanish health science libraries (\#AyudaBiblioteca), organized to collaborate and provide a better response to the information needs resulting from Covid-19. It has 90 participants, mainly from hospital libraries, as well as from research organizations, scientific societies, professional associations, ministries, universities, health technology evaluation agencies, and independent professionals. Whats $A p p$ has been used as a means of communication, and Google Drive as a document repository. Resources are displayed on a public website, and search strategies are deposited in Diigo. Twitter is used with the hashtags \#AyudaBiblioteca and \#COVID19 as an external means of communication to receive and disseminate information. This collaboration has allowed us to join forces in the search for and coordinate collection of reliable, evidence-based, proven, and high-quality information, demonstrating the benefits of teleworking and online collaboration. The benefits of open science and how it improves research are also reflected upon. This initiative shows the importance of health science librarians in supporting health, academic, and research staff.

\section{Keywords}

Health science libraries; Hospital libraries; Special libraries; Library cooperation; University libraries; Professional association libraries; Information centers; Librarians; Information specialists; Information professionals; Information scientists; Information dissemination; Library innovation; Remote working; Teleworking; Open access; Covid-19; Coronavirus; Pandemics.

\section{Introducción}

La pandemia causada por el coronavirus SARS-CoV2 ha provocado el cierre presencial de la mayoría de las bibliotecas. Sin embargo, éstas han seguido abiertas al público ofreciendo sus servicios de manera virtual y su personal continúa trabajando de forma activa, ofreciendo y mejorando sus servicios online. Este es el caso del colectivo que trabaja en las bibliotecas biomédicas en España que convocó una reunión virtual mediante Zoom a través de la lista de distribución $C 17^{2}$ el 16 de marzo de 2020, para compartir ideas y experiencias de teletrabajo. Durante el transcurso de la reunión se llegó a la conclusión de que la mayoría de los usuarios de las bibliotecas de Ciencias de la Salud iban a enfrentarse a los mismos problemas relacionados con la Covid-19 y, por tanto, tendrían las mismas necesidades informativas. Para aunar y reducir esfuerzos se propuso la colaboración entre esos bibliotecarios para proporcionar, en la medida de lo posible, información rigurosa y acreditada sobre Covid-19 que ayudara a sus usuarios en la toma de decisiones de manera rápida, efectiva y eficaz. En esa misma línea se expresaba la American Library Association:

"También aplaudimos los numerosos ejemplos de bibliotecarios que crean fuentes de información rápida y sirven a toda la comunidad en grupos de trabajo para ayudar con mensajes, recursos de información y la preparación de otras medidas. Sabemos que están haciendo mucho trabajo en su lugar en este momento, y su perspectiva y experiencia son invaluables" (ALA, 2020).

Esta necesidad de información relevante se une a la falta de tiempo para la búsqueda y localización por parte de los profesionales sanitarios, volcados enteramente en el cuidado de pacientes infectados.

Como establecen algunos autores (Featherstone et al., 2020; Ali; Gatiti, 2020; Morlas, 2020) la labor y habilidades de los bibliotecarios en la selección y diseminación de la información son cruciales como apoyo al personal sanitario y de investigación. Por ejemplo, tras el virus SARS de 2003, en referencia a los bibliotecarios de Ciencias de la Salud dicen:

"Las habilidades de los bibliotecarios para evaluar la información disponible resultaron cruciales para seleccionar evidencias de mejor calidad para apoyar la toma de decisiones administrativas. El análisis cualitativo resultó en una mayor comprensión de las necesidades de información sobre pandemias e identificó las mejores prácticas para diseminar información durante los períodos de alto estrés organizacional causado por la afluencia de nuevos casos de una enfermedad infecciosa desconocida" (Featherstone et al., 2020).

E incluso que

“...las bibliotecas deberían ser una parte integral de la estructura de preparación para emergencias de los hospitales y en el desarrollo de un protocolo en coordinación con los equipos de preparación para emergencias lo que permitiría un apoyo bibliotecario integrado" (Featherstone et al., 2020). 
También Ali y Gatiti (2020) afirman que son los bibliotecarios los que pueden apoyar al personal médico, académico y equipos de investigación en la identificación de los últimos estudios relevantes publicados en revistas científicas, bases de datos con acceso gratuito a artículos relacionados con la Covid-19, búsquedas bibliográficas, revisiones sistemáticas, etc.; o como afirma Lina Moses, epidemióloga y ecóloga de enfermedades en la Tulane School of Public Health and Tropical Medicine de la Tulane University (New Orleans), encargada de distribuir la información científica a los equipos de respuesta operativa de la OMS (Organización Mundial de la Salud) cuando cita las palabras de Elaine Hicks:

"Los bibliotecarios están en una posición única para participar en un rol de respuesta al utilizar sus conocimientos y habilidades" (citado en Morlas, 2020).

Ejemplos de la respuesta de las bibliotecas a esta pandemia son los servicios online que están ofreciendo iniciativas como las siguientes:

- “¿Cómo están reaccionando las bibliotecas universitarias de Rebiun ante la Covid-19?” de Rebiun (2020), que reúne en un único sitio la información y servicios que las bibliotecas universitarias españolas están prestando;

- las de muchas bibliotecas de hospitales en sus webs;

- "Covid-19 and the global library field" de IFLA, también traducida al español (IFLA, 2020), donde se detallan las circunstancias y características de bibliotecas de todo el mundo.

En general, todas ellas han ampliado los períodos de préstamo y han ofrecido servicios adaptados a las circunstancias (alfabetización informacional online, préstamo interbibliotecario, apoyo en actividades de investigación, respuestas por correo a las demandas de sus usuarios, negociación con editores para ampliar el acceso, aunque temporal, a todos los contenidos online, accesibilidad a recursos de educación médica) y están identificando e informando sobre recursos en acceso abierto. También seleccionan fuentes autorizadas, que estructuran y actualizan la información específica sobre Covid-19 a la vez que se están comunicando por redes sociales con sus usuarios, entre otros muchos servicios virtuales para apoyar el aprendizaje y la investigación. Así lo señalan los directores de varias bibliotecas estadounidenses (University of Michigan, Johns Hopkins University, Chicago, Yale, etc.):

"El cierre de los espacios físicos durante este tiempo resalta el papel fundamental de los servicios virtuales, los recursos y la formación ofrecida por las bibliotecas, y ha acelerado la mejora de muchos recursos y servicios remotos para apoyar y avanzar en la misión de investigación de las instituciones, la atención al paciente, la educación, innovación y salud pública" (Conte et al., 2020).

Excepcionalmente alguna biblioteca hospitalaria ha mantenido alguno de los servicios presenciales con limitaciones del número de profesionales presentes y de aforo, como el uso restringido de las salas de lectura a horarios reducidos, guardando distancias de seguridad entre usuarios o incluso cerrando directamente las salas de lectura. En otros casos el personal de la biblioteca ha compartido sus tareas propias con otras de apoyo a otros departamentos, y algunos bibliotecarios se han visto ubicados en otros espacios del hospital, lo que les obliga a dedicar parte de su tiempo libre a prestar el servicio de biblioteca.

También se han dado casos en los que las bibliotecas, una vez cerradas al público, se han remodelado para convertirlas de manera provisional en salas de hospitalización/UCl. Es el caso de las bibliotecas de:

- Hospital Germans Trias i Pujol (Badalona) (Morales, 2020);

- Hospital Gregorio Marañón (Madrid) (Europa Press, 2020);

- Hospital Príncipe de Asturias (Alcalá de Henares) (Valdés, 2020).

Son bibliotecas que tienen un alto valor asistencial y disponen de fondos impresos de publicaciones científicas que las convierten en referentes en el ámbito bibliotecario médico español.

La experiencia que están viviendo las bibliotecas puede ser también una oportunidad para aprender a afrontar futuras crisis en este mundo globalizado. Las bibliotecas y su personal, con una gran tradición de colaboración, pueden compartir conocimientos y buenas prácticas. Un ejemplo es la encuesta que fue contestada, en menos de un mes, por más de 800 bibliotecas académicas, de las más de 3.000 instituciones en EUA, sobre Covid-19. El objetivo del estudio realizado por Lisa-Janicke Hinchliffe y Christine Wolff-Eisenberg (véase Heckel, 2020) era facilitar a los bibliotecarios académicos la toma de decisiones informadas al comprender mejor el panorama de lo que estaba sucediendo. Los resultados se iban publicando en tiempo real en un portal para facilitar su difusión.

https://bit.ly/39UhCH7

La encuesta incluye preguntas sobre medidas de limpieza y seguridad de las bibliotecas, acceso a materiales impresos, políticas sobre empleados que trabajan en forma remota, etc.

El documento Turning the threat of Covid-19 into an opportunity for greater support to documentary heritage (Unesco, 2020) señala la oportunidad que esta crisis ofrece para preservar y permitir el acceso digital al patrimonio documental. En esa misma línea, puede ser un impulso a la ciencia abierta, aprovechando el despliegue de información sobre la Covid-19 que editoriales, instituciones y otros proveedores de información han ofrecido en abierto al público. También en el ámbito de la docencia para apoyar el aprendizaje y la enseñanza online, al menos mientras dure el período no presencial en las aulas debido al confinamiento. En otras palabras, y tal y como se indica en la Declaración a favor del conocimiento abierto y sostenible (CRUE, 2020), 
"la lucha contra la pandemia del Covid-19 ha puesto en evidencia la importancia de compartir conocimiento científico de forma inmediata y abierta entre la comunidad investigadora",

Y se urge

“...tanto a las instituciones que apoyan y realizan investigación como a los editores y proveedores de servicios de información a que aceleren la transición hacia el acceso abierto dentro de un marco de sostenibilidad económica".

Esta idea también es impulsada en el documento Data together. Covid-19 appeal and actions, que requiere la implementación acelerada del ecosistema de los principios FAIR.

https://codata.org/wp-content/uploads/2020/03/Data-Together-COVID-19-Statement-FINAL.pdf

En la misma línea hay que destacar el desarrollo de OpenAire Covid-19 Gateway y sus Resources

https://www.openaire.eu/openaire-covid-19-gateway

https://bit.ly/3k9WIIQ

que indica

"Se producirán diferentes tipos de datos de diferentes disciplinas para ser utilizados en la investigación y en decisión de políticas. Teniendo un único punto de acceso, una puerta, como resultado de la colaboración de datos y conocimientos, especialmente porque esto es impulsado por prácticas científicas abiertas".

Lo mismo puede decirse del repositorio Zenodo, con una comunidad de investigación sobre Covid-19 que recopila los resultados de investigación:

https://zenodo.org/communities/covid-19?page $=1 \&$ size $=20$

Asimismo hay que mencionar la carta abierta de la IFLA y sus socios: https://www.ifla.org/files/assets/hq/topics/exceptions-limitations/documents/200403_joint_letter_to_dr_frOancis_gurry.pdf

que ha sido firmada por más de 140 organizaciones e individuos, dirigida al director general de la Organización Mundial de la Propiedad Intelectual (OMPI), sobre la urgente necesidad de combatir la pandemia de la Covid-19 y sus consecuencias, subrayando que estando

"obligados a cerrar sus puertas al público, las bibliotecas deben poder proporcionar acceso en apoyo de la investigación, la educación y el acceso a la cultura a distancia. Sin esta posibilidad, no sólo es más difícil contrarrestar la propagación de la Covid-19, sino que hay una interrupción más generalizada que dejará cicatrices en el futuro.

Sin embargo, las leyes y prácticas de propiedad intelectual no necesariamente lo permiten. Si bien ha habido muchas iniciativas positivas de los editores, a menudo en respuesta a las llamadas de las bibliotecas, éstas no cubren todas las necesidades o situaciones".

El objetivo de este artículo es describir la experiencia surgida de un grupo de profesionales de bibliotecas de Ciencias de la Salud españolas. Este grupo autodenominado \#AyudaBiblioteca \#COVID19 nació de manera espontánea para colaborar y dar una mejor respuesta a las necesidades de información de los profesionales sanitarios relacionadas con la Covid-19 para hacer frente a la pandemia actual, y en general a la sociedad. El grupo está formado mayoritariamente por bibliotecas de hospitales, pero también de institutos de investigación, centros de investigación médica aplicada, sociedades científicas, colegios profesionales, universidades, agencias de evaluación de tecnologías sanitarias, centros de documentación, consejerías de salud y profesionales independientes.

\section{Método}

En un primer momento nadie del grupo \#AyudaBiblioteca \#COVID19 se planteó si era una iniciativa única, si había precedentes, ni si se podría aprender de lo que ya hubiesen hecho otras bibliotecas en situaciones semejantes, sino en ponerse directamente en acción, y continuar trabajando, aprovechando la apertura de recursos y mediando como gestores de información y conocimiento científico para ponerlos al servicio de los usuarios reales, potenciales o virtuales, como es habitual en el quehacer diario, para ayudar con su profesionalidad, experiencia y su saber hacer en la búsqueda de información que requiriesen sus usuarios o cualquier sanitario. La mayoría de los profesionales que empezaron participando en esta iniciativa conocían:

- los recursos de la ALA para bibliotecas ante la pandemia: http://www.ala.org/tools/atoz/pandemic-preparedness

- la declaración de ICOLC (Coalición Internacional de Consorcios de Bibliotecas) sobre la pandemia mundial Covid-19: https://icolc.net/statement/statement-global-covid-19-pandemic-and-its-impact-library-services-and-resources

- algunas webs de recursos que ya habían empezado a elaborarse sobre el tema, como la de la biblioteca del Instituto de Salud Carlos III y otras bibliotecas que poco a poco habían considerado prioritario tener informada a la comunidad de usuarios de su institución.

Pero gracias a la colaboración del grupo \#AyudaBiblioteca \#COVID19, se fueron compartiendo y conociendo otros proyectos, recursos, publicaciones en acceso abierto e iniciativas a nivel mundial:

- recopilación de LibGuides de las bibliotecas americanas sobre Covid-19:

https://bit.ly/3fr2KRN 
- estrategias de búsqueda temáticas (como las de las bibliotecas australianas): https://www.alia.org.au/groups/HLA/covid-19-live-literature-searches

- Nilde World, de las universidades y centros de investigación y biomédicos de Italia, para proporcionar acceso a la documentación científica indispensable no sólo para las actividades de investigación, estudio y enseñanza, sino también para actualizar a los profesionales que tratan la Covid-19:

https://nildeworld.bo.cnr.it/it/content/cbn

El proyecto de colaboración comenzó con la reunión virtual mencionada anteriormente en la que surgió la idea de crear un grupo de WhatsApp para ayudar a responder las dudas que los usuarios iban planteando a cada biblioteca. El grupo que se autodenominó \#AyudaBiblioteca \#COVID19 se creó ese mismo día 16 de marzo. Todos son administradores del mismo, pudiendo enviar el enlace para unirse a todos los compañeros de bibliotecas/centros de documentación de Ciencias de la Salud que deseen participar en la iniciativa de cooperación.

En el grupo de WhatsApp \#AyudaBiblioteca \#COVID19 es donde fluye la información desde las 8 de la mañana hasta altas horas de la noche, los 7 días de la semana, siendo cientos los mensajes diarios. Se ha creado en Google Drive una carpeta compartida en la que los participantes tienen derecho de edición. Los miembros del grupo organizan la diversa documentación e información, recopilan y distribuyen la misma en carpetas para que pueda ser utilizada por todos: artículos de opinión, cartas al editor, casos clínicos, consensos, editoriales, ensayos clínicos, guías, infografías, manuales, meta-análisis, artículos de investigación, preguntas basadas en evidencias, procedimientos, recomendaciones, revisiones narrativas, revisiones rápidas, revisiones sistemáti-

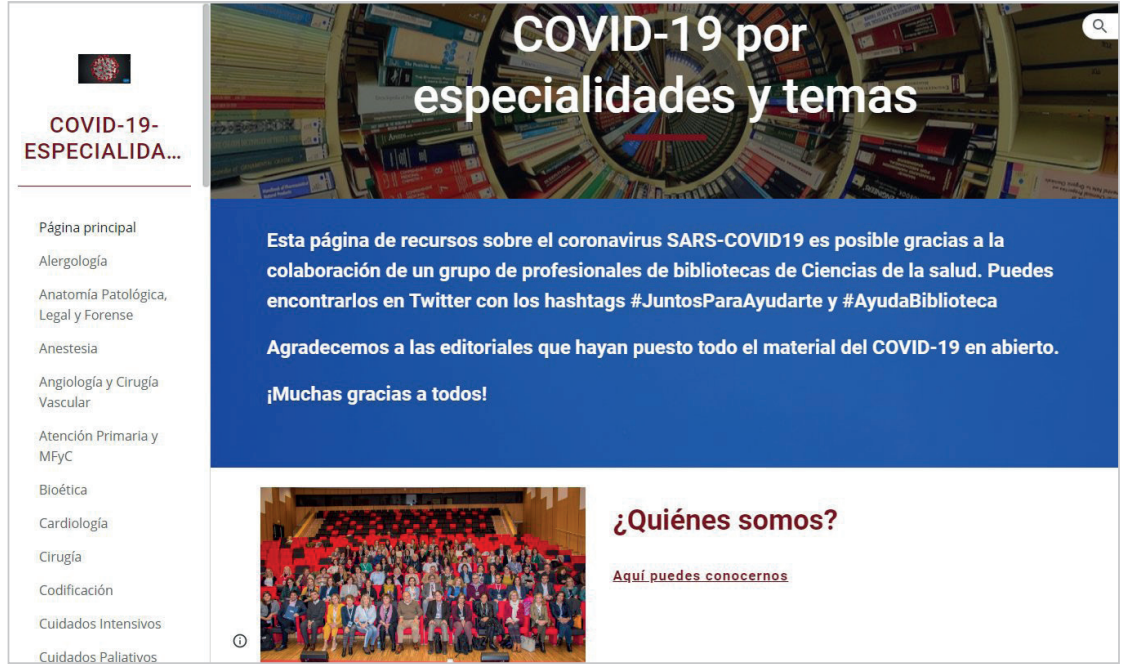

Figura 1. Covid-19 por especialidades y temas.

https://sites.google.com/view/covid19-por-especialidades

cas, series de casos e informes de casos, sumarios de evidencias y otros documentos. Esta documentación se visualiza y comparte en una web actualizada diariamente: Covid-19 por especialidades y temas (figura 1).

Se ha recopilado toda esta información a partir de fuentes de información de calidad, como bases de datos biomédicas reconocidas, evidencia clínica, revistas científicas y de investigación, sitios web confiables nacionales e internacionales, guías de recursos, fuentes de noticias y agencias oficiales, redes sociales y colegas profesionales sanitarios que hacían llegar propuestas de inclusión de documentos de su área de conocimiento (en ocasiones aportando incluso una lectura crítica de los mismos). Se han creado alertas, se evalúa la credibilidad de la información antes de difundirla y se incorpora al Drive compartido. Previamente se comprueba la existencia de duplicados y se consensua su ubicación, etc., utilizando como centro de comunicación el grupo \#AyudaBiblioteca \#COVID19 creado en WhatsApp. Cualquier miembro puede compartir las preguntas que le han llegado de sus usuarios y sus respuestas o preguntar dudas.

El 20 de marzo se creó un grupo público en Diigo, donde se comparten estrategias de búsqueda indicando la fuente y el creador de dicha estrategia. Es un sistema de gestión de información personal, que incluye marcadores web, bloc de notas, post-its, archivo de imágenes y documentos, así como selección de textos destacados (figura 2).

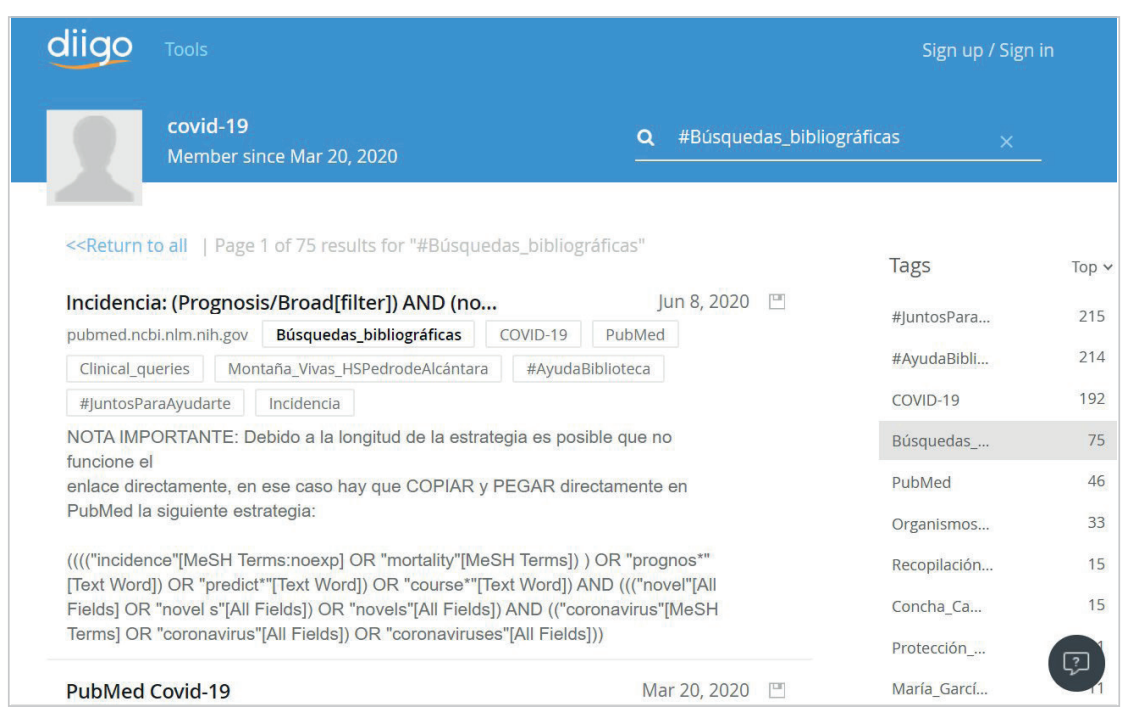

Figura 2. Página de Diigo mostrando una ecuación de búsqueda en PubMed.

https://www.diigo.com/profile/covid-19?query=\%23B\%C3\%BAsquedas_bibliogr\%C3\%A1ficas 


\begin{tabular}{|c|c|c|c|c|c|}
\hline MOST ACTIVE ? & Total tweets & RETWEETERS ? & Retweets & ORIGINAL TWEETS? & tweets \\
\hline $\begin{array}{l}\text { @biblioGetafe } \\
\text { Concepcion Campos-... }\end{array}$ & 70 & $\begin{array}{l}\text { @biblioGetafe } \\
\text { Concepcion Campos-... }\end{array}$ & 53 & $\begin{array}{l}\text { @biblioGetafe } \\
\text { Concepcion Campos-... }\end{array}$ & 17 \\
\hline $\begin{array}{l}\text { @bibliotecagaicr } \\
\text { BIBLIOTECA-GAI CREAL }\end{array}$ & 17 & $\begin{array}{l}\text { @bibliotecagaicr } \\
\text { BIBLIOTECA-GAI CREAL }\end{array}$ & 17 & $\begin{array}{l}\text { @Bibliosaude } \\
\text { Bibliosaude }\end{array}$ & 15 \\
\hline 2. @jmedino & 16 & $\begin{array}{l}\text { @jmedino } \\
\text { Juan Medino }\end{array}$ & 12 & $\begin{array}{l}\text { @Saludteca } \\
\text { Saludteca }\end{array}$ & 10 \\
\hline $\begin{array}{l}\text { @Bibliosaude } \\
\text { Bibliosaude }\end{array}$ & 16 & $\begin{array}{l}\text { @BiblioSalud_CYL } \\
\text { BiblioSalud_CYL }\end{array}$ & 11 & $\begin{array}{l}\text { @BiblioCHT } \\
\text { Biblioteca CHT }\end{array}$ & 7 \\
\hline C @Saludteca & 11 & Elena Primo & 9 & $\begin{array}{l}\text { @Xonxagg } \\
\text { Asun }\end{array}$ & 6 \\
\hline
\end{tabular}

Figura 3. Cuentas de Twitter que más han contribuido con el hasthag \#AyudaBiblioteca del 7 al 11 de abril de 2020. Se ha usado Binder.

Para resolver dudas o para unirse al grupo, se ha creado la cuenta de correo ayuda.biblioteca.ccss@gmail.com. Cuando se recibe una consulta, el grupo proporciona la mejor información posible a partir de fuentes de calidad y con suficiente evidencia. Siempre que se conozca la afiliación del usuario, se procura que sea su propia biblioteca quién le dé respuesta. A veces los usuarios trabajan en instituciones que no cuentan con el servicio de biblioteca especializada, en ese caso cualquiera de los integrantes del mismo puede contestar. Las respuestas también llevan los hashtags para que puedan ser localizados.

\section{Resultados}

Entre los resultados más destacables a fecha de 11 de abril de 2020 se podrían mencionar:

- Grupo de WhatsApp \#AyudaBiblioteca \#COVID19 en el que participan 90 profesionales de las bibliotecas de Ciencias de la Salud a nivel nacional y en el que se han compartido más de 1.000 archivos, enlaces y documentos.

- Drive compartido, con 70 carpetas y subcarpetas temáticas con más de 1.140 documentos, predominando los de tratamiento (102), protección del entorno laboral (81), radiología (63), y salud mental (65).

- Web Covid-19 por especialidades y áreas temáticas, desde alergología hasta virología (50 entradas distintas). En la denominación de las especialidades se ha tenido en cuenta la clasificación de las especialidades médicas oficiales y las sugerencias y recomendaciones directas de personal médico especializado.

- Recopilación en Diigo de más de 230 items, de los cuales 74 son estrategias de búsqueda realizadas en diferentes fuentes, mayoritariamente en PubMed, y 208 etiquetas (tags) de diferentes aspectos relacionados con la Covid-19.

- Presentación visual del grupo \#AyudaBiblioteca \#JuntosParaAyudarte \#COVID19 (en Genially), definiendo el proyecto, motivación, lo que realiza y cómo contactar.

https://view.genial.ly/5e92f5a5fba9d40d9e580bcb/horizontal-infographic-review-ayudabiblioteca

\begin{tabular}{|c|c|c|c|}
\hline MOST POPULAR ? & Followers & HIGHEST IMPACT ? & Impacts \\
\hline $\begin{array}{l}\text { @instCervantes } \\
\text { Instituto Cervantes }\end{array}$ & 213,114 & $\begin{array}{l}\text { @InstCervantes } \\
\text { Instituto Cervantes }\end{array}$ & 426,228 \\
\hline $\begin{array}{l}\text { @gobjccm } \\
\text { Castilla-La Mancha }\end{array}$ & 73,857 & $\begin{array}{l}\text { @biblioGetafe } \\
\text { Concepcion Campos-... }\end{array}$ & 140,630 \\
\hline $\begin{array}{l}\text { @joancmarch } \\
\text { joan carles march }\end{array}$ & 34,124 & $\begin{array}{l}\text { @gobjccm } \\
\text { Castilla-La Mancha }\end{array}$ & 73,857 \\
\hline $\begin{array}{l}\text {-1- @Bibliotecas_IC } \\
\text { Red de Bibliotecas del... }\end{array}$ & 28,718 & $\begin{array}{l}\text { @bibliovirtual } \\
\text { María bibliovirtual }\end{array}$ & 43,635 \\
\hline $\begin{array}{l}\text { @escpacientes } \\
\text { Escuela de Pacientes }\end{array}$ & 27,340 & $\begin{array}{l}\text { @soriano_p } \\
\text { Pedro Soriano \#Qued... }\end{array}$ & 39,794 \\
\hline
\end{tabular}

Figura 4. Cuentas de Twitter con más seguidores y con mayor impacto del 7 al 11 de abril de 2020. Se ha usado Binder. 
- Cuenta de correo electrónico ayuda.biblioteca.ccss@gmail. com, que ha recibido más de 100 consultas sobre cómo acceder a determinados documentos, solicitudes de información, peticiones de creación de carpetas de alguna especialidad, o para desdoblar alguna de las que ya existían, solicitudes para subir archivos y correos de agradecimiento... También se ha utilizado para recibir solicitudes de adhesión al grupo.

- Los bibliotecarios están recibiendo correos de agradecimiento del personal sanitario tras la información recibida, reconociendo el trabajo que realizan (figura 7), lo que está suponiendo un aumento en las peticiones de búsquedas bibliográficas y un aumento del conocimiento público del grupo.

- Se llevó a cabo una campaña en Twitter para las consultas de los profesionales sanitarios utilizando los hashtags \#Ayuda Biblioteca y \#COVID-19, \#JuntosParaAyudarte, \#BibliotecaEnCasa (utilizado por el Consejo de Cooperación Bibliotecaria) por un lado, para difundir la actividad del grupo, recomendando, por ejemplo, documentos de especial interés, y para recoger consultas de los profesionales sanitarios.

- Utilizando Binder se ha analizado el hashtag \#AyudaBiblioteca, obteniendo 500 tweets totales del 7 al 11 de abril, mostrando los siguientes datos: 235 usuarios han utilizado ese hashtag, con un impacto potencial de 1.236 .170 (número potencial de veces que alguien ha podido ver el hashtag \#AyudaBiblioteca). En la figura 3 se pueden ver las cuentas que más han contribuido en el tiempo analizado y en la figura 4, las cuentas con más seguidores y con mayor impacto del 7 al 11 de abril de 2020.

La visibilidad conseguida hasta el 13 de abril en medios de comunicación y redes sociales, fue la siguiente:

- 20 de marzo

Entrevista en LGN Radio a Isabel Martínez-Hervás (@MuCHaBiBLiO, Hospital Universitario Severo Ochoa) en el programa "Especial Héroes", donde habla de la iniciativa de las bibliotecas de ciencias de la salud \#AyudaBiblioteca con motivo del \#COVID19.

https://www.youtube.com/watch?time_continue=1\&v=kR8APIshZ6I\&feature=emb_logo

- 22 de marzo

“Colectivo de bibliotecarios de Ciencias de la Salud, 22 de marzo 2020". Desiderata. Biblioteconomía en España, n. 14, pág. 4, mayo-junio de 2020.

http://www.auxiliardebiblioteca.com/desiderata

- 31 de marzo

"Covid-19 y la colaboración espontánea de los profesionales de Ciencias de la Salud en España". Experiencia del colectivo contada por Maribel Domínguez-Aroca. Blog Universo Abierto.

https://universoabierto.org/2020/03/31/covid-19-yla-colaboracion-espontanea-de-los-profesionales-deciencias-de-la-salud-en-espana

- 31 de marzo

"\#AyudaBiblioteca, el personal bibliotecario de centros sanitarios se une para ofrecer información actualizada, fiable y de calidad". María García-Puente es entrevistada en el Blog de Julián Marquina.

https://www.julianmarquina.es/ayudabiblioteca-elpersonal-bibliotecario-de-centros-sanitarios-se-unepara-ofrecer-informacion-actualizada-fiable-y-decalidad

- 1 de abril

"Bibliotecas de hospitales unidas para compartir información sobre Covid-19". Diariofarma.

https://www.diariofarma.com/2020/04/01/bibliotecasde-hospitales-unidas-para-compartir-informacion-sobrecovid-19

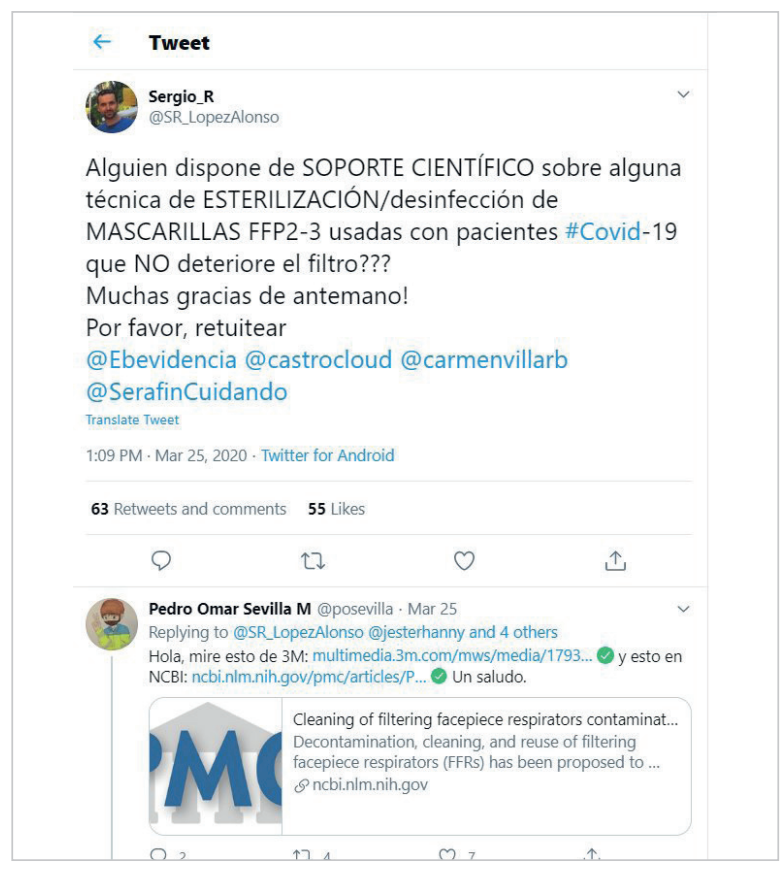

Figura 6. Ejemplo de información solicitada a \#AyudaBiblioteca y \#COVID19, y primera respuesta.

https://twitter.com/SR_LopezAlonso/status/1242770539220996096 
- 1 de abril

"La Biblioteca del Hospital Universitario de Asturias (HUCA) participa en un proyecto nacional de recopilación de información sobre la Covid-19". AsturSalud. Portal de Salud del Principado de Asturias.

https://www.astursalud.es/noticias/-/noticias/labiblioteca-del-huca-participa-en-un-proyecto-nacionalde-recopilacion-de-informacion-sobre-la-covid-19-

- 3 de abril

"Los hospitales de Alzira y Xàtiva participan en una red estatal de documentación sobre el coronavirus". Periódico Levante. El mercantil valenciano.

https://www.levante-emv.com/ribera/2020/04/03/ hospitales-alzira-xativa-participan-red/1997828.html

- 7 de abril

"Las bibliotecas de Ciencias de la Salud del Sescam participan en la iniciativa nacional para ofrecer información actualizada sobre COVID-19". Sescam. Consejería de Sanidad de Castilla La Mancha.

https://sanidad.castillalamancha.es/saladeprensa/ notas-de-prensa/las-bibliotecas-de-ciencias-de-lasalud-del-sescam-participan-en-la

- 7 de abril

"La Biblioteca del Hospital Universitario de Asturias (HUCA) recopila documentación sobre Covid-19". La nueva España, Sociedad, Noticias de Oviedo.

https://www.Ine.es/sociedad/2020/04/07/biblioteca-huca-recopila-documentacion-covid/2621915.html

- 7 de abril

"Las bibliotecas de Ciencias de la salud del Sescam ofrecen información actualizada sobre el Covid-19". Acta sanitaria. https://www.actasanitaria.com/bibliotecas-sescam-covid-19

- 7 de abril

"Las bibliotecas de Ciencias de la Salud del Sescam ofrecen información actualizada sobre el Covid-19". Objetivo Castilla-La Mancha noticias.

https://objetivocastillalamancha.es/contenidos/region/bibliotecas-ciencias-salud-sescam-participan-iniciativanacional-ofrecer-informacion-actualizada-sobre-covid-19

- 7 de abril

"Coronavirus. Las bibliotecas de Ciencias de la Salud del Sescam ofrecen información actualizada sobre el Covid19". El digital de Ciudad Real.

https://www.eldigitaldeciudadreal.com/2020/04/07/coronavirus-las-bibliotecas-de-ciencias-de-la-salud-del-sescamofrecen-informacion-actualizada-sobre-el-covid19

- 9 de abril

"Noticias: Las bibliotecarias de Sacyl colaboran en una iniciativa nacional de información sobre Covid-19". Sacyl. Junta de Castilla y León.

https://www.saludcastillayleon.es/institucion/es/biblioteca/noticias/bibliotecarias-sacyl-colaboran-iniciativanacional-informac

- 11 de abril

“Coronavirus en Ibiza. La biblioteca científica del Área de Salud pitiusa recopila información científica sobre el Covid-19. Diario de Ibiza.

https://www.diariodeibiza.es/pitiuses-balears/2020/04/11/coronavirus-en-ibiza-la-biblioteca/1136010.htm/

- 13 de abril

"Papel de las bibliotecas de hospital frente al Covid-19". Reportaje en España directo a Isabel Martínez Hervás, Biblioteca del Hospital Universitario Severo Ochoa.

https://www.youtube.com/watch?v=20tmtuF49Do\&feature=youtu.be

- Guías de recursos que mencionan como fuente de actualización las del grupo de bibliotecarias/os en Ciencias de la Salud \#AyudaBiblioteca \#COVID19. Entre otras (ver Anexo):

- Guía Covid-19, Biblioteca Nacional de Ciencias de la Salud (Instituto de Salud Carlos III);

- Biblioguía Covid-19. Biblioteca de la Universidad de Alcalá. 


\section{Discusión y conclusiones}

Se ha realizado una búsqueda bibliográfica para conocer la existencia de otras iniciativas parecidas de bibliotecas frente a una pandemia para saber si la experiencia que aquí se plasma de la colaboración espontánea de profesionales de bibliotecas de Ciencias de la Salud tenía de alguna forma precedente. Lo más aproximado podría encontrarse en la monografía de Robertson, que incluye un apartado sobre "Perspectiva de la pandemia: cómo un brote podría afectar a las bibliotecas" (apartado 27) donde indica:

"No sabemos cuándo estallará una pandemia. Es un ejercicio útil conside-

rar los efectos de una pandemia en las operaciones de la biblioteca. La gripe aviar podría estallar en Asia y extenderse por todo el mundo. Los suministros adecuados de vacunas podrían no estar disponibles. Las bibliotecas podrían cerrarse por largos períodos. El riesgo de vandalismo en las instalaciones de la biblioteca podría aumentar. Los despidos podrían ocurrir. Los recursos electrónicos podrían mantener las bibliotecas en funcionamiento" (Robertson, 2015).

En ese mismo texto se exponen las preocupaciones sobre los efectos de una pandemia en las bibliotecas y se enuncian algunas soluciones como, por ejemplo, un buen servicio de limpieza, como con el brote de SARS en Toronto en 2003, aconsejando que:

"Los bibliotecarios podrían adaptar varios procesos de servicio técnico para continuar de forma temporal con red inalámbrica, y los administradores de la biblioteca podrían reunirse y tomar decisiones fácilmente a través de un sistema inalámbrico como lo hacen a través de video llamadas".

Y se señala que en una pandemia las bibliotecas estarán obligadas a una respuesta de emergencia, como el cierre de los centros o las precauciones de volver al trabajo después de que la pandemia se haya calmado, pues muchos bibliotecarios, por seguridad preferirán seguir con servicios digitales.

Esta pandemia ha fomentado el teletrabajo en muchos sectores incluido el de las bibliotecas, práctica poco extendida en la Administración a la que pertenece la gran mayoría de los integrantes del grupo \#AyudaBiblioteca \#COVID19, demostrando la eficiencia con los resultados aquí expuestos. De esta situación también se concluye que el teletrabajo debería incorporarse a la práctica laboral de las bibliotecas, no en su totalidad, pero sí de forma estable.

Las bibliotecas de Ciencias de la Salud están bien posicionadas para responder a la crisis del Covid-19 en palabras de Lisa-Janicke Hinchliffe de la biblioteca de la University of Illinois at Urbana-Champaign:

"Las bibliotecas han tenido esta infraestructura digital en desarrollo durante las últimas dos décadas. Han estado trabajando simultáneamente en una biblioteca digital y en una biblioteca presencial. Me siento muy orgullosa de la comunidad de bibliotecas académicas y de cómo han protegido la seguridad del personal y los usuarios al cerrar las ubicaciones físicas, al tiempo que proporcionan un alto nivel de servicio y agregan algunos servicios cuando los miembros de la facultad y estudiantes cambiaron a online" (Heckel, 2020).

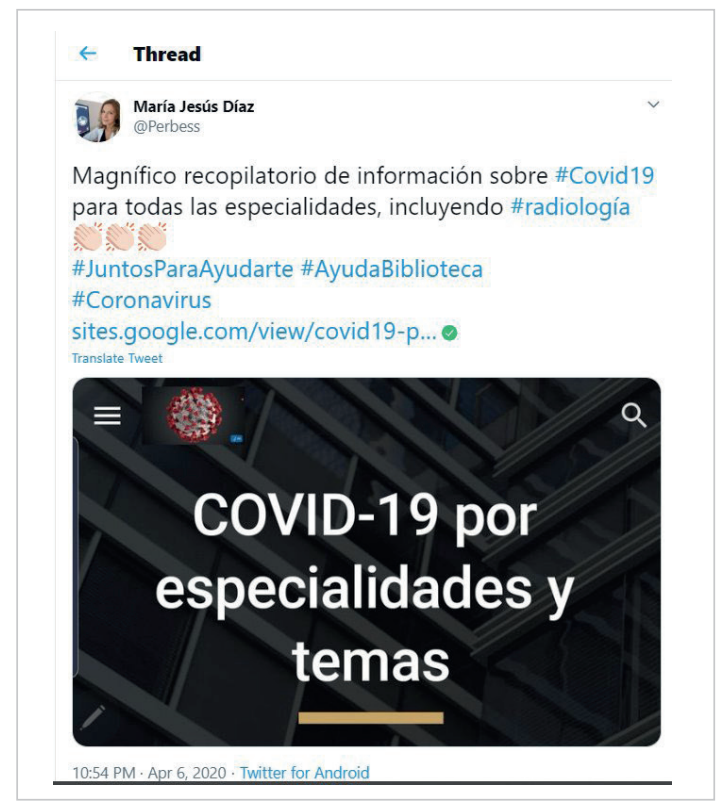

Figura 9. Ejemplo de reconocimiento al trabajo del grupo \#AyudaBiblioteca \#COVID19

https://twitter.com/Perbess/status/1247251305279696897 
Además, se ha constatado que las redes sociales como WhatsApp y Twitter ofrecen a los bibliotecarios unas plataformas idóneas para la colaboración y difusión de información rigurosa y acreditada sobre el Covid-19 para que los profesionales sanitarios puedan tomar decisiones informadas de fuentes confiables.

Por último, se vuelve a demostrar el espíritu de cooperación que las bibliotecas de Ciencias de la Salud siempre han tenido con miras a su fin último, servir a sus usuarios de la forma más eficiente posible. También se han constatado las dificultades de utilización las aplicaciones colaborativas, al exigir una coordinación constante y normalización que se ha ido desarrollando y armonizando según se ha visto la necesidad de hacerlo. Es de destacar que es la primera vez que las bibliotecas de Ciencias de la Salud han dado una respuesta conjunta y en tiempo real a los profesionales sanitarios.

Las bibliotecas continúan evolucionando y llevando a cabo nuevas soluciones para enfrentarse a los desafíos de la información, y este texto es un ejemplo más de cómo están respondiendo y aprendiendo ante la pandemia. En resumen, la acción que diariamente están llevando a cabo debe ser destacada y conocida por la sociedad. Profesionales, que como muchos otros están en un entorno de vida con mayores o niños que atender, familiares enfermos a quienes cuidar, recibiendo noticias de familiares, amigos o conocidos enfermos o que han fallecido, pero todos unidos colaborando con ilusión, solidaridad, responsabilidad, entusiasmo y mucha profesionalidad.

\section{Anexo}

Recursos e iniciativas públicas llevadas a cabo en bibliotecas especializadas en Ciencias de la Salud en España a raíz de la situación provocada por el coronavirus. También en algunos casos, los bibliotecarios mantienen información y recursos en la intranet de su institución, pero aquí sólo se recogen los accesibles por internet.

\section{Recursos ad hoc}

- BiblioSaúde: https://bibliosaude.sergas.gal/DCarruselPortada/116/Recursos\%20Covid19_gal250320.pdf

- Biblioteca Nacional de Ciencias de la Salud (ISCIII). Instituto de Salud Carlos III: https://www.isciii.es/QueHacemos/Servicios/Biblioteca/Paginas/Guia-COVID.aspx

- Biblioteca Sanitaria Online de Castilla y León: https://www.saludcastillayleon.es/institucion/es/biblioteca/recursos-evidencia-cientifica-covid-19

- Biblioteca Virtual del Sistema Sanitario Público de Andalucía: https://www.bvsspa.es/profesionales/noticia/recursos-biblograficos-COVID-19

- Biblioteca Virtual Sant Joan de Déu: https://bibliosjd.org/2020/03/24/covid-19-recursos-biblioteca-virtual

- Hospital Universitario Central de Asturias: https://bibliotecahuca.com/covid-19-2

- Hospital Universitario 12 de Octubre: https://coronavirusbiblioh12o.wordpress.com

- Hospital Universitario de Fuenlabrada: https://www.netvibes.com/bibliohflr\#Coronavirus_COVID19

- Hospital Universitario de Getafe: https://sites.google.com/view/informacion-covid-19/principal

- Hospital Universitario La Paz: https://proyectofcw20.wordpress.com/2020/03/14/covid-19-informacion-contrastada-y-recursos-web

- Hospital Universitario Puerta de Hierro: https://sites.google.com/site/bibliotecapuertahierro

- Hospital Universitario Virgen de las Nieves: https://www.huvn.es/investigacion/biblioteca_y_gestion_documental/covid_19

- MurciaSalud: http://www. murciasalud.es/pagina.php?id=458211\&idsec $=5$

- Portal del conocimiento. Coronavirus SSPA (Covid-19). Junta de Andalucía. Consejería de Salud y Familias. [Apoyo de los bibliotecarios en la recopilación de documentos y enlaces]: https://portalandaluciacovid19.es

- Universidad de Alcalá: https://uah-es.libguides.com/covid-19

- Universidad Complutense de Madrid: https://biblioguias.ucm.es/recursos-sobre-coronavirus 


\section{Blogs de seguimiento de la actualidad}

- BiblioGetafe:

https://ccamposhugf.wordpress.com

- BiblioHFLR:

https://bibliohflr.wordpress.com

- BiblioMadSalud: http://www.bibliomadsalud.com/blo

- Biblioteca Médica Virtual:

https://bibliovirtual.wordpress.com/novedades-y-recursos

- Píldoras. Blog de Saludteca. Biblioteca Online Sanitaria de Extremadura: https://mariamontanavivas.wordpress.com

\section{Notas}

1. Colaboradores del grupo de profesionales de Ciencias de la Salud \#AyudaBiblioteca \#COVID19 que han participado en este trabajo

\begin{tabular}{|c|c|c|}
\hline 1 & Almazán-Segovia, Elia & Documentalista especializada en Ciencias de la Salud \\
\hline 2 & Alonso Martín, Marisa & Biblioteca. Complejo Hospitalario de Toledo \\
\hline 3 & Ansuategi Zengotitabengoa, Eukene & Biblioteca OSI Donostialdea. Hospital Universitario Donostia \\
\hline 4 & Campal Robledo, Teresa & Biblioteca. Luciano Castañón. SESPA. Gijón \\
\hline 5 & Calvo Ferrer, Ana Elvira & Bibliosaúde. Biblioteca da Consellería de Sanidade \\
\hline 6 & Campos Asensio, Concepción & Biblioteca. Hospital Universitario de Getafe (Madrid) \\
\hline 7 & Castelló Zamora, Belén & Biblioteca. Gobierno Vasco. Departamento de Salud \\
\hline 8 & Catalán Vega, Marcos Antonio & Biblioteca. Parc Sanitari Sant Joan de Déu (Barcelona) \\
\hline 9 & Cibeira Badía, Amelia & Biblioteca. Complexo Hospitalario de Vigo - Hospital Meixoeiro \\
\hline 10 & Colmenares Brunet, Isabel & $\begin{array}{l}\text { Biblioteca Josep Laporte. Fundació Salut i Envelliment. Casa Convalescència. Universitat Autò- } \\
\text { noma de Barcelona }\end{array}$ \\
\hline 11 & Costa Marín, Maria & Biblioteca. Hospital Can Misses (Ibiza) - Bibliosalut \\
\hline 12 & De Andrés Loste, Cuca & Biblioteca. Hospital Universitario Río Hortega (Valladolid) \\
\hline 13 & De la Cámara De las Heras, Juanma & Biblioteca. Hospital Universitario La Ribera. Alzira (Valencia) \\
\hline 14 & Díaz Ruiz, María Pilar & Biblioteca. Consejería de Sanidad/Gerencia Regional de Salud. Castilla y León \\
\hline 15 & Escudero Gómez, Cristina & Biblioteca. Hospital Puerta de Hierro (Madrid) \\
\hline 16 & Esteve Esteve, Gemma & Biblioteca. Hospital Lluís Alcanyís (Xàtiva, Valencia) \\
\hline 17 & Estrada Lorenzo, José Manuel & Biblioteca. Hospital 12 de Octubre \\
\hline 18 & Fernández Gómez, Manuel & Biblioteca Ciencias de la Salud. Universidad de Oviedo \\
\hline 19 & Fernández Luque, Antonia María & Biblioteca. Hospital de la Axarquía \\
\hline 20 & Galán Grajera, María Eulalia & Biblioteca. Hospital Universitario del Henares \\
\hline 21 & García García, Asunción & Biblioteca. Escuela Universitaria de Enfermería Cruz Roja (Madrid) \\
\hline 22 & García-Puente, María & Biblioteca. Hospital Fundación Jiménez Díaz. AlterBiblio (Madrid) \\
\hline 23 & Gómez Sánchez, Alicia Fátima & Consultora independiente \\
\hline 24 & González-Larragán, Susana & Biblioteca. OSI Ezkerraldea Enkarterri Cruces. Hospital Universitario Cruces (Vizcaya) \\
\hline 25 & González Fresneda, Amparo & Biblioteca. Hospital Universitario Virgen de las Nieves (Granada) \\
\hline 26 & Gudín Uriel, Cecilia & Gudinfo. Gestión del conocimiento \\
\hline 27 & Guerra Rodríguez, Mercedes & Biblioteca. Sociedad Española de Reumatología (Madrid) \\
\hline 28 & Gutiérrez Casado, Natalia & Hospital Universitario del Sureste (Madrid) \\
\hline 29 & Gutiérrez Couto, Uxía & Biblioteca. Complexo Hospitalario Universitario de Ferrol. Bibliosaúde \\
\hline
\end{tabular}




\begin{tabular}{|c|c|c|}
\hline 30 & Herrera Ramos, Estefanía & $\begin{array}{l}\text { Profesional independiente (Documentalista del Sescs en excedencia desde } 2019 \text { (Agencia } \\
\text { Evaluación de Tecnologías Sanitarias) }\end{array}$ \\
\hline 31 & Higueras Callejón, Camila & Biblioteca. Escuela Andaluza de Salud Pública (Granada) \\
\hline 32 & Isabel Gómez, Rebeca & Biblioteca. AETSA, Evaluación de Tecnologías Sanitarias de Andalucía \\
\hline 33 & Jarillo Pizarro, Alicia & Biblioteca. Hospital Fundación Jiménez Díaz \\
\hline 34 & Jiménez Varas, Miguel Ángel & Biblioteca. Hospital Universitario La Paz \\
\hline 35 & Lejona Martínez de Lecea, Begoña & Biblioteca. OSI Bilbao-Basurto. Hospital Universitario Basurto. Osakidetza \\
\hline 36 & Marquínez Cabrejas, María & Biblioteca. Universidad de Navarra. Clínica-Cima UNAV \\
\hline 37 & Martínez-Hervás, Isabel & Biblioteca. Hospital Universitario Severo Ochoa \\
\hline 38 & Medino Muñoz, Juan & Biblioteca. Hospital Universitario de Fuenlabrada \\
\hline 39 & Merino Márquez, Ana María & Biblioteca Sant Joan de Déu (Barcelona) \\
\hline 40 & Mones Iglesias, Ángel Luis & Hospital Álvarez-Buylla, Mieres (Asturias) \\
\hline 41 & Mota Perera, María Luisa & Biblioteca. Complejo Hospitalario Universitario de Badajoz \\
\hline 42 & Muñoz Martín, Beatriz & Biblioteca. Compejo Asisencial de Zamora. Sacyl \\
\hline 43 & Osorio Calles, Francisco Javier & Biblioteca. Complexo Hospitalario Universitario Pontevedra \\
\hline 44 & Palop Valverde, María & Biblioteca. Hospital General Universitario de Ciudad Real \\
\hline 45 & Pinín Osorio, Carolina & Biblioteca. Hospital Universitario Central de Asturias \\
\hline 46 & Primo Peña, Elena & Biblioteca Nacional de Ciencias de la Salud, ISCIII \\
\hline 47 & Rebollo Rodríguez, María José & Biblioteca. Colegio de Médicos de Madrid \\
\hline 48 & Redondo García, José Carlos & Biblioteca Virtual de la Consejería de Sanidad de la Comunidad de Madrid \\
\hline 49 & Reillo Sánchez, Olga & Biblioteca. Hospital Universitario Infanta Sofía \\
\hline 50 & Rivas Ramos, Catalina & Biblioteca. Hospital de Motril (Granada) \\
\hline 51 & Roca Bonals, Anna & Biblioteca. Facultat de Medicina i d'Infermeria. Universitat de Girona \\
\hline 52 & Rodríguez Otero, Carmen & Biblioteca. Bibliosaúde. Biblioteca Virtual do Sistema Sanitario Público de Galicia \\
\hline 53 & Rodríguez Vera, Isabel & Biblioteca. Complejo Hospitalario Universitario de Albacete \\
\hline 54 & Salcedo Sánchez, M. Carmen & Biblioteca. Hospital de Montilla (Córdoba) \\
\hline 55 & Sastre Suárez, Silvia & Gerencia de Atención Primaria de Mallorca. Bibliosalut (Baleares) \\
\hline 56 & San José Montano, Blanca & Biblioteca. Hospital Universitario de Móstoles \\
\hline 57 & Sánchez Sánchez, Blanca & Biblioteca. Hospital de la Vega Lorenzo Guirao, Cieza (Murcia) \\
\hline 58 & Sanmartín Reinoso, Yolanda & Biblioteca. CHUVI. Hospital Álvaro Cunqueiro, Vigo \\
\hline 59 & Toledano Redondo, Salvador & Biblioteca. Hospital Universitario Reina Sofía (Córdoba) \\
\hline 60 & Trigueros Terrés, Rosa & Biblioteca. Hospital General Universitario de Alicante \\
\hline 61 & Ubeda Carrillo, Marimar & Biblioteca. OSI Donostialdea. Hospital Universitario Donostia \\
\hline 62 & Valencia Romero, Consuelo & Biblioteca. Hospital Virgen de la Luz (Cuenca) \\
\hline 63 & Villalba Díaz, Arantxa & Biblioteca. Hospital de Montilla (Córdoba) \\
\hline 64 & Vivas Jiménez, Montaña & Biblioteca. Complejo Hospitalario Universitario de Cáceres \\
\hline
\end{tabular}

2. C17 es un catálogo colectivo de publicaciones de Ciencias de la Salud, en el que participan alrededor de 500 bibliotecas especializadas en salud españolas. Su primera edición fue en cd-rom en 1996. En la actualidad su gestión y consulta se realiza online. Las bibliotecas participantes pueden consultarlo gratuitamente, pero existen consultas avanzadas de pago. 


\section{Bibliografía}

ALA (2020). "ALA executive director Tracie Hall releases statement on coronavirus". ALA news, 14 March.

http://www.ala.org/news/press-releases/2020/03/ala-executive-director-tracie-hall-releases-statement-covid-19

Ali, Muhammad-Yousuf; Gatiti, Peter (2020). "The Covid-19 (coronavirus) pandemic: reflections on the roles of librarians an information professionals". Health information and libraries journal, 6 April.

https://doi.org/10.1111/hir.12307

Arroyo-Almaraz, Isidoro; Calle-Mendoza, Samuel; Van-Wyk, Cliff (2018): “La eficacia en la comunicación de las ONGD. El uso de Facebook en campañas de emergencia". Revista latina de comunicación social, n. 73, pp. 765-789.

https://doi.org/10.4185/RLCS-2018-1281

Conte, Marisa; Gallagher, John; Holmes, Kristi L.; Jaguszewski, Janice M.; Kern, Barbara; Rethlefsen, Melissa L.; Seymour, Anne K. (2020). "Answering the call: academic health sciences libraries and Covid-19". NLM musings from the mezzanine: innovations in health information from the director of the National Library of Medicine, 7 April.

https://nImdirector.n/m.nih.gov/2020/04/07/answering-the-call-academic-health-sciences-libraries-and-covid-19

CRUE (2020). Declaración a favor del conocimiento abierto y sostenible. CRUE; Bucle; Bugalicia; CBUA; CSIC, CSUC; G9; Consorcio Madroño; Uniris.

https://www.rebiun.org/sites/default/files/2020-03/Declaracionafavordelconocimientoabiertoysostenible.pdf

Europa Press (2020). "El Gregorio Marañón reconvierte su biblioteca en una UCI con 19 camas". Europa Press, 4 abril.

https://www.europapress.es/madrid/noticia-hospital-gregorio-maranon-reconvierte-biblioteca-uci-19camas-20200404122841.html

Featherstone, Robin M.; Boldt, R. Gabriel; Torabi, Nazi; Konrad, Shauna-Lee (2012). "Provision of pandemic disease information by health sciences librarians: a multisite comparative case series". Journal of the Medical Library Association, v. 100, n. 2 , pp. $104-112$

https://doi.org/10.3163/1536-5050.100.2.008

Heckel, Jodi (2020). "Survey of US academis libraries documents Covid-19 pandemic responses". Illinois news bureau. Research news, 27 March.

https://news.illinois.edu/view/6367/807539

IFLA (2020). COVID-19 y el sector bibliotecario global. Federación Internacional de Asociaciones de Bibliotecarios y Bibliotecas.

https://www.ifla.org/ES/node/92983

Morales, Agus (2020). "Metamorfosis de un hospital". Revista 5W, 2 abril.

https://www.revista5w.com/why/metamorfosis-un-hospital

Morlas, Amanda (2020). "Tulane University librarians help provide critical information to WHO teams". Tulane news, 13 April.

https://news.tulane.edu/pr/tulane-university-librarians-help-provide-critical-information-who-teams.

Rebiun (2020). “¿Cómo están reaccionando las bibliotecas universitarias de Rebiun ante el Covid-19?”. Rebiun, marzo. https://www.rebiun.org/noticias/universidades/covid-19

Robertson, Guy (2015). Robertson on library security and disaster planning. Amsterdam: Chandos Publishing. ISBN: 978 0081000885

Unesco (2020). Turning the threat of Covid-19 into an opportunity for greater support to documentary heritage. https://en.unesco.org/sites/default/files/dhe-covid-19-unesco_statement_en.pdf

Valdés, Isabel (2020). "Las urgencias 'de guerra' del Hospital de Alcalá". El país, 30 marzo.

https://elpais.com/espana/madrid/2020-03-29/las-urgencias-de-guerra-del-hospital-de-alcala.html

Profesional de la

-información http://www.elprofesionaldelainformacion.com
Bienvenido a EP|

Revista cientifica internacional 